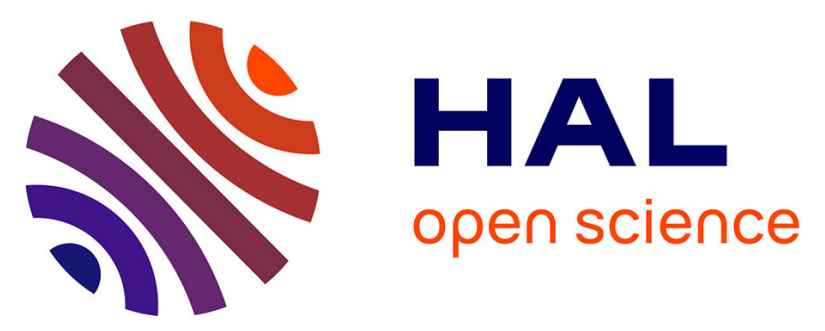

\title{
La démocratisation des contenus d'enseignement à l'école publique en Pologne: essai d'analyse des justifications politiques des réformes scolaires à la sortie du communisme.
}

Sébastien Urbanski

\section{To cite this version:}

Sébastien Urbanski. La démocratisation des contenus d'enseignement à l'école publique en Pologne: essai d'analyse des justifications politiques des réformes scolaires à la sortie du communisme.. Education et Sociétés : Revue internationale de sociologie de l'éducation, 2011, Redistribution, reconnaissance..justice?, 27, pp.147-160. 10.3917/es.027.0147 . halshs-01488033

\section{HAL Id: halshs-01488033 \\ https://shs.hal.science/halshs-01488033}

Submitted on 13 Mar 2017

HAL is a multi-disciplinary open access archive for the deposit and dissemination of scientific research documents, whether they are published or not. The documents may come from teaching and research institutions in France or abroad, or from public or private research centers.
L'archive ouverte pluridisciplinaire HAL, est destinée au dépôt et à la diffusion de documents scientifiques de niveau recherche, publiés ou non, émanant des établissements d'enseignement et de recherche français ou étrangers, des laboratoires publics ou privés. 


\title{
La démocratisation des contenus d'enseignement à l'école publique en Pologne :
} essai d'analyse des justifications politiques des réformes scolaires à la sortie du communisme.

\author{
Sébastien Urbanski
}

\begin{abstract}
Résumé : En Pologne, en 1989, le monopole étatique dans la définition des contenus d'enseignement a été aboli. Cependant, la très grande liberté donnée aux auteurs des manuels et des programmes scolaires est aujourd'hui partiellement remise en question par de nombreux pédagogues. Cette liberté pose en particulier un problème de laïcité, dans la mesure où elle permet l'intrusion de croyances personnelles - et notamment religieuses - dans ce qui est, officiellement, un enseignement universel. Dans cet article, nous essayons d'expliquer l'émergence de la loi sur l'école (1991) qui est à l'origine de ces modalités de conception des contenus d'enseignement, en montrant notamment qu'elle a été adoptée par les députés sur la base d'une convergence entre la critique marchande et la critique domestique de l'ancien système scolaire. Nous essayons également d'identifier des ressources argumentatives identitaires, dont la présence est explicable par le processus de décomposition du mouvement social Solidarité.
\end{abstract}

Dans les pays d'Europe centrale et orientale, après l'effondrement des régimes autoritaires du bloc de l'Est, les réformes scolaires ont été conçues, adoptées et mises en oeuvre très rapidement : en général, ce fut l'affaire de quelques mois, un an au plus. Dans un ouvrage sur la décentralisation de l'éducation dans les pays ex-communistes, I. Bajomi note que l'observateur pourrait être, dans un premier temps, impressionné par cette rapidité. Néanmoins, cette dernière pourrait très bien n'être finalement que de la précipitation à l'origine de nombreux problèmes (Bajomi 2002, 23). En Pologne, de nombreux spécialistes affirment qu'un problème majeur aujourd'hui est, entre autres, l'incohérence des programmes et des manuels, ainsi que la multiplication anarchique de ces derniers, due notamment à la très grande liberté donnée à leurs auteurs (Rott 2003 ; Konarzewski 2004 ; Kawalec 2008).

D'un autre côté, le monopole étatique dans la définition de ce qui doit être enseigné a bel et bien été aboli. Certes, dans certains domaines, le monopole d'État a été pour une bonne part remplacé par le pouvoir de l'Église catholique : par exemple, de nombreuses décisions concernant l'école sont prises dans le cadre de la «Commission du Gouvernement et de l'Épiscopat» (Komisja Wspólna Rządu i Espiskopatu). Le Ministre de l'Éducation a ainsi récemment affirmé que la décision concernant la possibilité de passer un examen de « religion » au baccalauréat n'était pas de son ressort, et qu'elle sera prise dans le cadre de cette commission (Gazeta Wyborcza 2008). Il reste, 
néanmoins, que la procédure de conception des programmes et des manuels scolaires est assez libérale.

En fait, il semble qu'un piège majeur ait été évité : l'enseignement n'est pas directement sous la tutelle idéologique de ces puissantes institutions potentiellement liberticides que sont l'État et l'Église. Mais en contrepartie, la grande liberté accordée aux auteurs des manuels scolaires est à l'origine de leur multiplication anarchique, souvent aux dépens de leur qualité. En outre, cette liberté se traduit parfois par l'intrusion de croyances personnelles dans ce qui est, officiellement, un enseignement universel. Pour en rester au problème de la laïcité qui est extrêmement aigu en Pologne, il n'est pas rare, par exemple, de trouver dans les manuels d'histoire des expressions du type «Après la mort et la résurrection de Jésus Christ, les apôtres... » (Pietracha 2001); ou des titres de chapitre fortement connotés métaphysiquement (par exemple «Jusqu'à la fin du monde » (Małkowski 2000) pour un chapitre consacré aux débuts du christianisme); ou encore des majuscules systématiques pour chaque occurrence d'un pronom désignant Jésus (Mrozewicz 2002), comme cela est pratiqué dans les manuels de catéchisme. C'est un genre de problème auquel les responsables de tout système éducatif sont confrontés d'une manière ou d'une autre : quel est le degré de liberté qu'il faut laisser aux auteurs des programmes et des manuels afin d'empêcher un contrôle idéologique, tout en assurant néanmoins un minimum de contrôle pédagogique ?

Dans cet article, nous voudrions étudier les justifications politiques qui ont été à l'origine des modalités contemporaines de la conception des programmes et des manuels scolaires polonais. L'analyse portera surtout sur les années 1989-1991, car c'est pendant cette période que la plupart des textes légaux importants sur l'école ont été conçus et adoptés - notamment la loi sur l'école de 1991, qui concerne l'enseignement public et privé (mais nous concentrerons notre étude sur l'enseignement public, notamment parce que l'enseignement privé n'est fréquenté en Pologne que par 2\% des élèves - voir Putkiewicz et Wiłkomirska 2004). Bien entendu, il est possible d'identifier d'importantes réformes scolaires dans les années suivantes, mais il reste que les modalités institutionnelles générales de la conception des programmes et des manuels scolaires n'ont pas beaucoup changé depuis 1991. La démarche de ce qui suit est la suivante : nous prendrons pour point de départ certaines remarques générales contenues dans un ouvrage en français sur la décentralisation dans les pays ex-communistes de l'Europe centrale et orientale (Bajomi et Derouet 2002), d'une part pour préciser leur pertinence sur le cas polonais, et d'autre part pour les intégrer dans une théorie sociologique attentive à ces constantes de l'argumentation que sont les lieux communs ou topoï (Bouvier 2005). Les questions qui sous-tendent cette étude sont les suivantes : comment a-t-il été possible, d'un point de vue politique, de libéraliser les procédures de conception 
des programmes et des manuels scolaires polonais, et quelles sont les implications de cette libéralisation?

Nous essaierons de montrer qu'il est possible de trouver des éléments de réponse du côté des topoï argumentatifs qui ont permis notamment aux ministres, aux députés et aux syndicats de justifier leurs croyances et leurs actions dans un contexte de transition vers un capitalisme de type occidental. De ce point de vue, les topoï de validité très générale repérés par Boltanski et Chiapello (1999) dans le processus de justification du capitalisme nous sont apparus précieux. Mais, dans l'oeuvre de ces auteurs, l'aspect qui retiendra prioritairement notre attention est le repérage des réservoirs d'arguments (les « cités ») et non l'aspect pragmatique (les « épreuves »). C'est la raison pour laquelle nous utiliserons le terme de topos (topoï au pluriel), au sens de lieu commun, pour dénoter ces réservoirs d'arguments (pour des travaux de sociologie de l'éducation mettant prioritairement l'accent sur les lieux communs, voir par exemple Derouet-Besson 2004).

Mais nous identifierons également la place de ressources argumentatives identitaires, dans la mesure où les forces politiques au principe de la libéralisation de l'école étaient issues d'un mouvement social (Solidarność ou Solidarité) qui nécessitait précisément la constitution - ou au moins la reconnaissance subjective - d'une identité collective (Touraine et al. 1982). Les ressources argumentatives identitaires ont de ce fait été centrales, comme nous essaierons de le montrer, dans la période en question.

Préalablement, il est nécessaire de présenter les principaux acteurs politiques présents à la sortie du communisme. L'installation au gouvernement, en 1989, des leaders du mouvement social Solidarité a ouvert une période - à certains égards toujours actuelle - dans laquelle se sont affrontées, schématiquement, trois grandes forces politiques :

- Le camp post-communiste (dont les représentants au Parlement se définissaient principalement comme social-démocrates) qui disposait de ressources institutionnelles fortes (comme par exemple un syndicat enseignant fortement implanté au niveau local) mais de ressources argumentatives identitaires faibles (du fait de leur appartenance passée à un régime très largement considéré comme oppresseur) ;

- Le camp de Solidarité (dont les représentants au Parlement se sont regroupés dans le Club Parlementaire Citoyen ou OKP - Obywatelski Klub Parlamentarny) lui même subdivisé en deux camps comprenant :

- d'une part, ceux qui s'identifiaient au mouvement Solidarité dans sa composante antiétatique et donc potentiellement anti-gouvernementale ; 
- d'autre part, ceux qui reconnaissaient la légitimité du gouvernement Solidarité.

Le tableau suivant résume les points forts et les points faibles de chaque camp, ainsi que les conceptions affichées concernant l'école :

\begin{tabular}{|c|c|c|c|c|}
\hline & $\begin{array}{l}\text { Ressources } \\
\text { institutionnelles } \\
\text { particulièrement } \\
\text { développées }\end{array}$ & $\begin{array}{l}\text { Ressources } \\
\text { argumentatives }\end{array}$ & $\begin{array}{l}\text { Rapport au } \\
\text { mouvement Solidarité }\end{array}$ & Quelle école? \\
\hline $\begin{array}{l}\text { 1. Solidarité } \\
\text { anti-étatique }\end{array}$ & $\begin{array}{l}\text { Syndicat Solidarité : } \\
\text { peu de pouvoir } \\
\text { concret mais fort } \\
\text { pouvoir symbolique } \\
\text { (Wałęsa, porté par L. } \\
\text { Kaczyński) }\end{array}$ & $\begin{array}{l}\text { Fortes au niveau } \\
\text { identitaire : c'est le } \\
\text { Syndicat qui dispose } \\
\text { en droit du logo } \\
\text { Solidarité }\end{array}$ & $\begin{array}{l}\text { Positif (essai de } \\
\text { stimulation du } \\
\text { mouvement par } \\
\text { l'intermédiaire de fortes } \\
\text { personnalités comme } \\
\text { Kaczyński ou Wałęsa) }\end{array}$ & $\begin{array}{l}\text { Tant que la révolution de } \\
\text { Solidarité destinée à éliminer } \\
\text { les communistes n'a pas eu } \\
\text { lieu, une école d'État n'a guère } \\
\text { de légitimité ; celle-ci doit } \\
\text { donc être au service des } \\
\text { parents ; elle ne peut être } \\
\text { laïque }\end{array}$ \\
\hline $\begin{array}{l}\text { 2. Solidarité } \\
\text { pro- } \\
\text { gouvernement }\end{array}$ & $\begin{array}{l}\text { Gouvernement } \\
\text { (Mazowiecki) et } \\
\text { direction de l'OKP } \\
\text { (Geremek) }\end{array}$ & $\begin{array}{l}\text { Fortes : les pro- } \\
\text { gouvernement sont } \\
\text { surtout des } \\
\text { intellectuels par } \\
\text { ailleurs fortement } \\
\text { liés aux intellectuels } \\
\text { occidentaux }\end{array}$ & $\begin{array}{l}\text { Méfiant (en raison de } \\
\text { l'aspect anti-étatique du } \\
\text { mouvement) }\end{array}$ & $\begin{array}{l}\text { L'école doit s'adapter aux } \\
\text { réalités économiques ; } \\
\text { cependant elle doit rester } \\
\text { laïque }\end{array}$ \\
\hline $\begin{array}{l}\text { 3. Post- } \\
\text { communistes }\end{array}$ & $\begin{array}{l}\text { Appareil d'État (dont } \\
\text { les rectorats, les } \\
\text { écoles...) }\end{array}$ & $\begin{array}{l}\text { Faibles en raison de } \\
\text { la filiation } \\
\text { communiste } \\
\text { (Kwaśniewski a } \\
\text { demandé pardon } \\
\text { plusieurs fois) }\end{array}$ & $\begin{array}{l}\text { Négatif mais } \\
\text { respectueux (dans la } \\
\text { mesure où le } \\
\text { mouvement Solidarité } \\
\text { a une dimension } \\
\text { nationale) }\end{array}$ & $\begin{array}{l}\text { École d'État, laïque et } \\
\text { officiellement égalitaire (mais } \\
\text { l'école communiste était } \\
\text { fortement inégalitaire, } \\
\text { notamment selon une } \\
\text { opposition ville/campagne) }\end{array}$ \\
\hline
\end{tabular}

Tableau 1 : points forts, points faibles et conceptions de l'école des principaux camps politiques après la

formation du gouvernement Mazowiecki

Aujourd'hui, l'école polonaise n'est pas laïque, n'est pas au service des parents ; elle est officiellement égalitaire, et s'est accommodée aux recommandations de l'O.C.D.E. en matière d'adaptation économique du système éducatif (Bajomi 2002, 22). Étant données les rivalités entre ces trois camps, comment expliquer cette évolution?

À notre connaissance, aucune étude approfondie n'a été réalisée sur le thème de la démocratisation des contenus d'enseignement à l'école publique en Pologne. La dimension empirique de ce qui suit est donc basée principalement sur des sources de première main, et en particulier sur les archives des discussions de la Table Ronde, du Parlement et des revues officielles des deux plus grands syndicats enseignants polonais. 


\section{La dynamique des topoï argumentatifs justifiant les politiques scolaires au fur et à mesure du processus de décomposition de Solidarité}

La première remarque générale sur laquelle nous prendrons appui est empruntée à G. Halasz :

Les coalitions qui appuyaient la décentralisation étaient souvent des coalitions politiques négatives, c'està-dire unies par un rejet commun de l'ancien système plutôt que par le désir de progresser ensemble vers un objectif partagé. Du coup, ces coalitions très fragiles n'ont duré que le temps du démantèlement et n'ont pas été capable de définir les axes de la reconstruction (Halasz 2002, 175)

Le terme de coalition politique négative, utilisé ici par Halasz, est important pour notre propos. Nous essaierons en effet de montrer que le caractère très libéral des programmes et des manuels scolaires a eu son origine dans l'aspect négatif des coalitions politiques polonaises des années 1989 à 1991. Selon nous, celui-ci se manifestait dans une large mesure par l'insistance des premiers élus de la Pologne démocratique sur l'opposition binaire entre « eux » (les communistes) et « nous » (Solidarité). Une telle démarcation identitaire était elle même basée sur des mécanismes institutionnels concrets, qui sont les suivants : la très forte hiérarchisation, avant 1989, du pouvoir communiste mais aussi de l'opposition anti-communiste (Rykowski 1994); et les contrats dits « de la Table Ronde » entre les portes-parole de l'opposition et les portes-parole des communistes (Elster 1996).

Lors des accords de la Table Ronde (et donc avant la formation du gouvernement Mazowiecki), il n'y avait encore que deux groupes d'acteurs en présence: Solidarité et les communistes. Le topos servant à justifier les réformes scolaires était alors clairement de type inspiré. C'est ainsi que les communistes et Solidarité sont parvenus à se mettre d'accord :

\section{J. Fisiak, Ministre communiste de l'Éducation}

«L'école a une fonction créative », elle doit « donner une chance d'autoréalisation pour des enseignants créatifs », elle doit «éveiller l'invention des groupes sociaux»;
H. Samsonowicz, Représentant de la Commission à l'Éducation du syndicat Solidarité

«A l'école, il doit y avoir de la place pour l'homme excentrique, pour l'homme original, pour l'homme qui a ses intérêts, ses opinions »; « il s'agit de donner (...) aux 
l'éducation «se réalisera avec de l'engagement et de la créativité » (Nowa Szkoła 1989, pp. 397-398) enfants de plus grandes possibilités de cultiver leurs (...) tendances naturelles et leurs intérêts » (ibidem, p. 404408)

$\underline{\text { Tableau } 2 \text { : Exemples de justification des axes des politiques scolaires lors des accords de la Table Ronde }}$

À cette époque (la première moitié de l'année 1989), il n'existait pas de dissensions visibles au sein du camp de Solidarité, que l'on peut encore qualifier de mouvement social organisé contre un État dominateur. Mais une fois que l'élite du mouvement prit place, en août 1989, au sein du gouvernement Solidarité (dont le chef était Mazowiecki), les dissensions ont commencé à apparaître. À vrai dire, il y a toujours eu de très forts désaccords au sein du mouvement: l'apparence d'unité de celui-ci, fortement véhiculée par les médias occidentaux, était due à sa très forte hiérarchisation (Wedel 1989). Quoi qu'il en soit, une fois que les candidats de Solidarité eurent gagné les élections, un nouveau rapport de forces argumentatif se mit en place entre non plus deux, mais principalement trois forces politiques : le gouvernement Solidarité d'où les réformes scolaires furent pilotées, les ex-communistes et ceux qui, méfiants envers le nouveau gouvernement, déclaraient rester fidèles à l'idée anti-étatique de ce qui restait du mouvement social Solidarité (voir tableau 1).

Ainsi, dès la publication de la première ébauche de la nouvelle loi sur l'école, le Ministère de l'Éducation (camp 2 du tableau 1) fut la cible de critiques émanant du syndicat enseignant Solidarité (camp 1 du tableau 1). Selon les représentants de celui-ci, la loi ne reconnaissait pas assez les compétences des « corps sociaux », et en particulier celles des parents. Les arguments à l'appui de cette thèse ne mettaient plus l'accent sur la créativité, comme dans la phase précédente dans laquelle le camp de Solidarité manifestait encore une unité, mais clairement sur la famille. Il ne s'agit pas, croyons-nous, d'un simple glissement sémantique, mais bien d'un changement de régime de justification, comme le suggère l'échange suivant issu de la première rencontre - après la publication de l'ébauche de loi - entre le Ministère de l'Éducation (représenté ici par la vice-ministre A. Radziwiłl, AR) et le syndicat enseignant Solidarité (représenté par L. Kujawski, LK) :

LK : Mais l'école (...) éduque sur l'autorisation des parents, pas le contraire.

AR : Pas complètement, parce qu'elle n'est pas un gouverneur.

LK : Elle ne l'est pas, mais elle agit au nom des parents.

AR : Mais aussi au nom de la nation toute entière (...).

LK : La soumission de l'éducation à des buts nationaux, comme nous le savons d'après l'histoire [référence à la période communiste - l'auteur], peut-être dangereuse.

AR : Non, ce n'est pas une soumission (...). 
LK : Nous avons fait l'expérience de déviations du système scolaire [référence à la période communiste l'auteur], c'est pour cela que nous voulons la démocratie et le primat des parents (Przegląd Oświatowy (PO) $1 / 6 / 1990)$

Kujawski semble ici considérer que les parents constituent la source de légitimité exclusive de l'école. Radziwiłł voit dans la «nation» une autre source de légitimité. D'un point de vue politique plus général, les deux protagonistes se reconnaissent dans Solidarité, mais le syndicaliste peine à reconnaître la représentativité du gouvernement: pour lui, Solidarité est d'abord un mouvement, basé sur un syndicat (voir tableau 1). C'est peut-être pour cela qu'il fait ici référence, deux fois, à la période communiste durant laquelle les ministres étaient largement considérés comme non-représentatifs. La vice-ministre admet au contraire la représentativité du gouvernement, notamment parce qu'elle en fait partie. Pour elle, apparemment, la présence de membres de Solidarité au gouvernement suffit à en faire un gouvernement représentatif (malgré la présence d'excommunistes dans l'appareil d'État). Ainsi, deux topos (un topos domestique et un topos civique) butent l'un contre l'autre sans qu'un accord puisse être trouvé. Nous verrons plus loin (partie II) que malgré la persistance de ce désaccord, l'adoption de la loi sur l'école s'est réalisée sur la base d'une convergence entre le topos domestique (soutenu par le mouvement Solidarité) et le topos marchand (soutenu par le gouvernement Solidarité), et plus précisément sur la base d'une critique de l'État que des arguments basés sur ces topoï permettent.

Une deuxième ressource argumentative générale des représentants du syndicat enseignant était de continuer à considérer Solidarité comme un mouvement d'opposition à l'État (ce qui était par ailleurs délicat puisqu'une partie du mouvement avait déjà pris place au sein du gouvernement). Ainsi, un représentant du syndicat, J. Rybicki, reprocha au Ministre de l'Éducation H. Samsonowicz de considérer que les engagements de la Table Ronde sont toujours valides : « ne fallait-il pas, dès l'automne [1989], radicaliser ces accords qui n'étaient plus actuels ? (PO 15/5/1990). Le Ministre, au contraire, s'appuyait précisément sur ces derniers pour légitimer son action ; il répondit en effet : «quand on s'engage à accomplir une action donnée, on ne peut pas changer ses idées sous l'influence des évènements présents » (id.). Précisons que la question de savoir si les accords de la Table Ronde sont actuels était d'une importance cruciale pour tous ceux qui s'identifiaient à Solidarité : fallait-il en effet continuer à soutenir le mouvement (avec sa composante anti-étatique) ou fallait-il soutenir le gouvernement Solidarité dont la plupart des membres a conclu les accords de la Table Ronde? L'importance de cette question explique par ailleurs la fréquence avec laquelle l'argument de la (non-)légitimité de ces accords - et celui, connexe, de la (non-)légitimité du gouvernement - a été utilisé pendant cette période. 
Ce passage, pour ceux qui s'identifiaient au mouvement, de l'insistance sur la créativité à l'insistance sur la famille peut être expliqué par le processus de décomposition de Solidarité : pendant les discussions de la Table Ronde, une «poignée d'hommes » (Wedel, art. cit.) organisés autour d'un syndicat exprimait la croyance collective d'un large groupe unifié principalement sur la base de son opposition au pouvoir des communistes ; mais, après les élections de juin 1989, l'appui institutionnel de ceux qui se réclamaient de Solidarité n'était plus constitué uniquement par le syndicat du même nom, mais aussi par un gouvernement. Les croyances de ceux qui s'identifiaient à Solidarité ne pouvaient donc que difficilement demeurer unifiées. Dans ce cadre, la justification prenait appui sur des topoï généraux, mais aussi sur un capital social identitaire que le gouvernement et le syndicat se disputaient, le problème étant dans ce second cas de savoir qui est ou qui représente Solidarité. Ce deuxième type de justification prenait parfois une tournure assez subtile. Par exemple, voici comment le secrétaire d'État à l'éducation W. Kulerski tentait, face aux critiques du syndicat enseignant, de fonder sa légitimité à prendre des décisions :

\footnotetext{
Nous avons le syndicat Solidarité [il s'agit ici du syndicat d'avant juin 1989 - l'auteur], ses représentants ont été désignés pour former un gouvernement, ne pouvons-nous donc pas nous identifier à « Solidarité » ? Est-ce que « Solidarité » est obligée d'être dans l'opposition ? (id.)
}

Ici, W. Kulerski essaie de situer son gouvernement dans sa lignée syndicale pour suggérer une certaine unité du camp de Solidarité. Mais il le fait en répondant en fait au même syndicat dans sa dimension institutionnelle - que celui dont il parle dans la phrase citée, et auquel il s'identifie. Cela illustre la progressive disjonction entre les croyances de ceux qui s'identifiaient à Solidarité et la base institutionnelle initiale du mouvement (constituée par le syndicat d'avant juin 1989). Cette disjonction s'accompagna d'un changement de régime de justification chez tous ceux qui s'identifiaient à Solidarité. Nous voudrions exposer maintenant certaines conséquences de ces transformations.

\section{La diminution du rôle de l'État après 1989 : les places respectives des critiques domestique, marchande et identitaire de l'ancien système scolaire}

Une deuxième remarque d'ordre général sur laquelle nous basons notre propos est empruntée à I. Bajomi qui note, à propos de la décentralisation dans les pays autrefois communistes, que « la redistribution des compétences est destinée à augmenter l'adaptabilité du système éducatif, mais ce 
type d'évolution peut aussi être interprété comme une forme concrète du désengagement de l'État » (Bajomi 2002, 23). En Pologne, ceux qui revendiquaient l'interprétation du désengagement de l'État étaient les membres - ex-communistes - du plus grand syndicat enseignant (l'Association de l'Enseignement Polonais ou ZNP - Związek Nauczycielstwa Polskiego) ainsi que la plupart des députés ex-communistes réunis dans le puissant Club Parlementaire de la Gauche Démocratique (PKLD - Klub Parlamentarny Lewicy Demokratycznej), qui tentaient de freiner la redistribution des compétences. Mais, comme nous essaierons de le montrer, celle-ci put néanmoins dans une large mesure se réaliser sur la base d'une convergence entre deux critiques de l'ancien système scolaire : la critique domestique portée entre autres par le syndicat enseignant Solidarité, et la critique marchande portée par le gouvernement qui se qualifiait de «libéral» et dont un souci majeur était de se conformer à la thérapie de choc conçue par J. Sachs, économiste américain et conseiller au FMI et à la Banque Mondiale (nous prenons ici appui sur des hypothèses que Van Zanten et Ball (2000) développent sur l'exemple de l'Angleterre).

Face au problème du transfert des compétences en matière de conception des programmes, la vice-ministre de l'Éducation réduisit ces derniers et décida de laisser aux enseignants le soin de décider du contenu de la leçon correspondant au temps ainsi libéré. Il s'agissait donc d'un programme minimum central assorti éventuellement - selon les ressources de l'établissement - d'un programme complémentaire local. Par ailleurs, la première version de la loi sur l'école prévoyait que le Ministère «suggèrera » (et non «autorisera ») l'utilisation de tel ou tel manuel scolaire (Nowa Szkola 1990). L'intention première était donc une très forte libéralisation pédagogique. Cette renonciation partielle au contrôle pédagogique devait, selon le projet de loi, s'accompagner d'une renonciation de l'obligation étatique de financer directement les écoles primaires et les collèges (qui devaient passer sous la responsabilité des communes, distinctes de l'État). Ce projet rencontra d'abord une résistance très forte de la part du principal syndicat enseignant ZNP (ex-communiste), dont les critiques étaient surtout de type social et civique :

\section{Critiques de type social :}

«Le Ministère essaie de proposer, dès le niveau élémentaire, des écoles pour les pauvres et pour les riches » (Głos Nauczycielski (GN) 2/4/1991). «Dire que les communes devront initier leurs 'propres actions' pour trouver de l'argent, c'est raconter des histoires » (GN 9/5/1991).

\section{Critiques de type civique :}

«Le Ministre de l'éducation ne peut pas renoncer à avoir une influence sur l'école (...). Les corps sociaux ne peuvent avoir plus de compétences que n'en ont les pouvoirs éducatifs plus qualifiés » (GN 20/5/1991).

Tableau 3 : Exemples de critiques du syndicat ZNP 
L'aspect civique des arguments utilisés (dans le sens où le Ministère est considéré comme représentatif) est très probablement explicable par la présence de fonctionnaires ex-communistes dans l'appareil d'État (voir tableau 1) : il est compréhensible que ceux-ci ne veuillent pas renoncer à leur position et donc au contrôle étatique de l'école. Dans ce cadre, l'idée selon laquelle le Ministère n'est en charge que d'un programme minimum central était accueillie avec un grand scepticisme : «Sans cours qui compléteraient et approfondiraient un tel programme 'minimisé', les chances éducatives des enfants sont quasi-nulles. C'est donc la fin de l'éducation gratuite... » (GN, 2/4/1991).

La critique du syndicat enseignant Solidarité était quant à elle, comme on l'a vu, principalement de type domestique; dans ce cadre, le projet de loi était perçu comme trop centralisé. Pour les membres de ce syndicat, il était envisageable que chaque école puisse élaborer intégralement son propre programme (PO, 1/4/1991). Par ailleurs, le pouvoir des directeurs d'école était considéré comme illégitime, contrairement au pouvoir que devraient avoir, entre autres, les parents :

«Et où est le rôle des enseignants dans l'évaluation [des enseignants] ? Et le rôle des parents ? Et le rôle des élèves ? Alors quoi, c'est encore le directeur qui va arbitrer secrètement l'évaluation ? [cette dernière phrase fait référence à la période communiste - l'auteur] » (PO, 8/4/1990).

Cette critique de la centralisation était par ailleurs fortement liée à la critique des fonctionnaires ex-communistes toujours en place : selon le syndicat, le gouvernement était inapte à mener la bataille pour la démocratie au niveau local parce qu'il se montrait réticent à les congédier, et parce que ce n'est qu'en les congédiant que la « révolution de Solidarité » aurait pu avoir lieu (PO, 15/4/91). Le centralisme du nouveau projet de loi sur l'école fut alors expliqué par le fait que « ce sont les fonctionnaires [ex-communistes] du Ministère qui l'ont écrit » (PO, 8/4/1990); ceux-ci furent d'ailleurs qualifiés de « nomenklatura » (id.).

Mais cette critique domestique (à laquelle le gouvernement répondait, comme nous l'avons suggéré dans la partie I, par des arguments de type civique) a partiellement convergé avec la critique marchande, mise en oeuvre par le gouvernement, de l'ancien système scolaire. Rivales sur la scène publique, ces deux critiques s'influençaient en coulisse mutuellement, et constituaient les deux bases sur lesquelles les politiques éducatives de redistribution des compétences se sont appuyées (nous paraphrasons ici Van Zanten et Ball 2000, 118). Le Ministre de l'Éducation n'a ainsi pas été contredit par le syndicat enseignant Solidarité lorsqu'il a exposé, lors d'une rencontre avec ce 
dernier, une conception marchande de la décentralisation du système scolaire basée sur l'idée que les problèmes financiers des écoles doivent être résolus par un renforcement des compétences économiques des communes, ainsi que par un affaiblissement parallèle de la responsabilité financière de l'État (PO, 1/2/1991). Par ailleurs, le syndicat enseignant Solidarité, au contraire de son rival ZNP, ne voyait pas de problème majeur dans l'injonction faite aux communes de trouver par elles-mêmes des moyens de financement, d'autant plus que cette injonction était propice à l'élimination des communistes encore en place dans l'administration scolaire (et donc à la « révolution de Solidarité »). Par exemple, L. Kujawski affirma que

dans la situation financière difficile que nous vivons en ce moment à propos de l'école, il faut trouver des moyens courageux et non-conventionnels d'obtenir de l'argent. De telles actions ne sauraient être réalisées par des cadres dirigeants routinisés, qui sont toujours en majorité de lignée nomenklaturale (PO, $1 / 5 / 1991)$.

Pendant ce temps, le syndicat ZNP mettait en oeuvre une critique civique qui renvoyait à une responsabilité exclusive de l'État. Son désaccord avec la politique gouvernementale était donc très grand, ce qui l'amena à émettre un vote de désaveu. Par ailleurs, il proposa au syndicat Solidarité de mener conjointement de sérieuses actions de protestation, mais ce dernier répondit que

\footnotetext{
ZNP [était] l'un des principaux collaborateurs de l'ancien régime communiste (...). ZNP est principalement le syndicat de l'ancienne nomenklatura, il suffit de lire Głos Nauczycielski pour s'en convaincre. Là, une occasion s'est présentée pour se montrer dans une autre lumière, dont on [ZNP] a avarement profité (PO, 1/10/1991)
}

Le syndicat Solidarité semblait ici suggérer que vu l'héritage communiste de ZNP, les protestations de celui-ci étaient a priori non crédibles. Le Ministre de l'Éducation soutenait des arguments similaires. Ainsi, le syndicat ex-communiste manquait de ressources argumentatives identitaires pour concrétiser son désaccord.

Il y a donc eu une certaine congruence locale entre la critique marchande mise en oeuvre par le gouvernement Solidarité et la critique domestique mise en oeuvre par ceux, députés ou syndicalistes, qui s'identifiaient encore au mouvement Solidarité dans sa version anti-étatique. Cette congruence s'explique par l'enjeu principal des réformes scolaires de l'époque : la diminution du rôle de l'État. Les topoï domestique et marchand sont en effet tous deux en contradiction potentielle 
avec l'anonymat que suppose le topos civique, et dont l'État peut être considéré comme une manifestation (Boltanski et Thévenot 1991). Par ailleurs, il semble que la critique domestique ait été considérablement renforcée par les ressources argumentatives identitaires dont le syndicat enseignant Solidarité disposait, et qui lui donnaient la possibilité d'accuser assez violemment, et crédiblement, les fonctionnaires d'État (qui étaient pour la plupart d'anciens communistes).

Bien entendu, et pour reprendre la terminologie de Van Zanten et Ball, il n'y pas eu que des accommodations nationales à la pression des logiques globalisantes : on peut aussi repérer certaines résistances. En particulier, le gouvernement prévoyait de considérer les dépenses de fonctionnement des écoles comme des dépenses privées, et donc soumises au régime d'imposition général; néanmoins, les députés ont modifié l'article en question dans le sens d'une exonération d'impôts. Cette décision s'est réalisée malgré la forte protestation du Ministre des Finances L. Balcerowicz en charge de mettre en oeuvre la thérapie de choc de J. Sachs (Archiwum Sejmu (AS), 18/7/1991).

\section{L'inscription dans la loi du système de valeurs chrétien : un compromis entre topos domestique et topos civique malgré la divergence entre les deux ailes de Solidarité ?}

Notre analyse des débats parlementaires confirme cette association, chez ceux qui s'identifiaient au mouvement, du topos domestique avec une critique des fonctionnaires excommunistes ainsi qu'avec une mise en doute de la légitimité de l'État (dont le gouvernement était considéré comme pas assez révolutionnaire). Nous proposons de considérer ici le cas de trois députés proches de L. Kaczyński - qui n'a pas cessé de soutenir, jusqu'à sa mort récente, l'idée d'une révolution de Solidarité (camp 1 du tableau 1).

Le député Twaróg affirma qu'«à l'école, l'occupation dure toujours» (il s'agit de l'occupation communiste) ; puis mit fortement en doute la légitimité de l'État : « si l'administration doit contrôler la réalisation des programmes scolaires (...), pourquoi diable adoptons-nous cette loi ?» (AS, 18/4/1991). Une critique de type domestique fut alors mise en oeuvre :

c'est sûrement pour cela que le projet de loi oblige les parents à assurer les conditions de scolarité des enfants. Bref, apparemment on considère encore que l'école va seulement donner des devoirs à la maison et poser des questions sans aucune responsabilité pour ses actions (id.).

Malgré ce type d'opposition ferme, le projet de loi fut adopté par les députés. Nous ne 
prétendons pas connaître les raisons précises de cette adoption; néanmoins, nous souhaiterions attirer l'attention du lecteur sur une proposition parlementaire qui a sans doute été d'un poids non négligeable dans l'apaisement des tensions entre les deux ailes de Solidarité (notées 1 et 2 dans le tableau 1) : celle de l'inscription dans la loi du « système de valeurs chrétien ». Cet élément peut en effet être interprété (par le sociologue et par les acteurs) comme un compromis entre le topos domestique et le topos civique, dans la mesure où il s'agit - par le biais d'un parlement considéré comme représentatif - de mentionner dans la loi des valeurs communes à la plupart des Polonais et ayant en l'occurrence un contenu domestique très important (Grabowska 2008). Ainsi, le député Czeżyk, au moment de déposer cette proposition de modification de la loi et après avoir indiqué qu'il existe un danger de "continuation du système d'indoctrination du socialisme réel », affirma qu'il faut «conférer un rôle à la famille» parce qu'« il est inacceptable que la famille n'ait pas d'influence sur ses enfants, dont l'esprit et les émotions ne sont pas encore formées, et qui ne voient pas encore complètement le monde de la connaissance à travers la croyance » (AS, 18/7/1991). Le discours du député Szatybełko était semblable, en ce qu'il faisait référence à la famille, au système de valeur chrétien et la persistance de l'idéologie communiste :

Sans Dieu, sans une famille morale et en bonne santé, c'était une idée [référence à l'idée communiste en général - l'auteur] digne du plus grand mensonge. Quand on lit le projet de loi sur le système scolaire, on voit ce courant mal dissimulé de cette idéologie anti-humaine, qui est encore un autre trait placé entre les paragraphes (id.)

La dernière proposition, et plus précisément le terme «trait», est très clairement une critique polémique de la politique dite du gros trait (grubej kreski) proclamée par Mazowiecki à son arrivée au gouvernement. Cette politique a largement été interprétée comme une volonté de tirer un « gros trait»sur les fautes passées des communistes, ce qui était difficilement acceptable pour la majorité de ceux qui continuaient à considérer Solidarité comme un mouvement social organisé contre un État dominateur. Le « trait placé entre les paragraphes » suggère ainsi l'alliance supposée du gouvernement Solidarité avec les ex-communistes. Plus généralement, il s'agit ici d'un durcissement de la position du camp 1 par rapport au camp 2 (tableau 1), lequel durcissement se manifeste encore aujourd'hui à travers le puissant parti Droit et Justice fondé par Kaczyński. Finalement, malgré l'opposition affichée de la majorité des ex-communistes et des membres du gouvernement, la mention du système de valeurs chrétien fut adoptée à trois voix près sur 256 (AS, 19/7/1991). 


\section{Les conséquences de la nouvelle loi sur l'école en matière de conception des programmes et des manuels scolaires}

L'adoption de la nouvelle loi sur l'école a permis de mettre sur pied un système d'enseignement non soumis directement à la pression idéologique de l'État ou de l'Église, du moins si l'on écarte le problème des croix dans les écoles et celui de l'inscription d'office des élèves aux cours de catéchisme. Cependant, comme nous l'avons signalé en introduction, la très grande liberté donnée aux auteurs des manuels a conduit à leur multiplication anarchique, souvent aux dépens de leur qualité. Cette situation peut être expliquée par les modalités de la redistribution des compétences éducatives menée au début des années 1990. Comme nous l'avons vu, le Ministre de l'Éducation a choisi de changer radicalement le principe top-down de conception des programmes et des manuels en un principe dans une large mesure bottom-up, c'est-à-dire accordant un grand pouvoir d'initiative à l'échelon local. Le temps a néanmoins manqué pour élaborer de nouveaux "programmes minimum centraux » cohérents (Konarzewski 2004, 13). Le seul contrôle des manuels mis en place a consisté à les soumettre à l'opinion de spécialistes de la discipline enseignée, et dans une large mesure choisis par l'auteur du manuel. Cela n'est pas suffisant pour limiter la prolifération de croyances personnelles - et notamment religieuses - dans l'enseignement public, d'une part parce que ces spécialistes peuvent partager les mêmes croyances personnelles en question, et d'autre part parce que de nombreux historiens reconnus en Pologne ne séparent pas leurs croyances religieuses de leurs travaux académiques (par exemple Jaczynowska (2006) qui par ailleurs supervise les manuels auprès du Ministère). Plus généralement, on peut mesurer ici la pertinence des remarques d' I. Bajomi et G. Halasz, qui mentionnent tous deux la précipitation dans laquelle se sont réalisées les réformes scolaires du début des années 1990 en Europe de l'Est.

Bien entendu, notre étude ne consiste pas à affirmer que les réformes scolaires de 1991 étaient «mal» conçues : un autre type de réforme aurait très probablement engendré d'autres problèmes. La tâche du Ministère de l'Éducation de l'époque était évidemment très ardue, puisqu'il s'agissait de rien de moins que d'instaurer durablement une forme de démocratie dans l'école. En ce qui concerne la question des programmes et des manuels, le Ministère était, semble-t-il, face à un dilemme : soit libéraliser radicalement le contenu des enseignements et par là rompre sérieusement avec le monopole étatique, avec cependant le risque d'une anarchisation des manuels scolaires ; soit mener des réformes plus progressives et peut-être plus cohérentes, avec cependant le risque de ne pas réellement libéraliser le contenu des enseignements scolaires et de ne pas remettre en cause le 
monopole étatique.

Aujourd'hui, un problème pédagogique central en Pologne consiste à essayer de renforcer le contrôle des manuels sans pour autant les remettre sous le quadrillage idéologique de l'État. Différentes tentatives de mainmise ministérielle sur les contenus enseignés ont été en effet récemment menées, à commencer par celle de R. Giertych (extrême droite) qui essaya de bloquer des manuels qui ne correspondaient pas, selon lui, à la «tradition polonaise » (Pezda 2007). On peut mentionner aussi le fait que le ministère social-démocrate au pouvoir de 2001 à 2004 semble avoir contesté le droit d'un de ses spécialistes à critiquer publiquement une réforme de centralisation de la conception des manuels (lettre du Ministère à destination de D. Rott, que ce dernier m'a aimablement communiquée).

Nous souhaiterions encore ajouter une remarque qui concerne la laïcité. Généralement, il semble que l'un des premiers problèmes qui vienne à l'esprit quand on parle de la laïcité scolaire polonaise est celui des cours de catéchisme (auxquels les élèves sont souvent inscrits d'office, même s'ils peuvent, en principe, en être dispensés). Nous espérons avoir mis l'accent sur un autre problème, qui à notre connaissance n'a pas du tout été abordé (même en Pologne) : celui de la présence de croyances religieuses personnelles dans les manuels de matières obligatoires (notamment l'histoire). Nous pensons que d'un certain point de vue, c'est un problème plus aigu que celui des cours de catéchisme, dans la mesure où aucun élève ne peut, bien évidemment, être dispensé du cours d'histoire (en France, ceux qui souhaitent prendre davantage en compte le « fait religieux » (Debray 2002) dans les matières obligatoires sont également confrontés à ce type de difficulté).

D'un point de vue plus général, nous espérons avoir montré comment différentes critiques du système scolaire polonais se sont complétées et influencées au début des années 1990, et avoir contribué par là, modestement, à une histoire des lieux communs utilisés pour justifier certaines politiques scolaires à l'origine de la démocratisation des contenus d'enseignement à l'école publique polonaise.

\section{Bibliographie}

Archiwum Sejmu Rzeczpospolitej Polskiej (Archives du Sejm de la République de Pologne), 18/4/1991, 18/7/1991, 19/7/1991, Warszawa, Wyd. Sejmowe.

BAJOMI, I. et DEROUET, J.-L. (dir.) 2002, La grande récréation. La décentralisation de l'éducation dans six pays autrefois communistes, Lyon, INRP. 

(2002).

BAJOMI, I. 2002, Points communs et divergences, in BAJOMI, I. et DEROUET, J.-L.

BOLTANSKI, L. et THEVENOT, L. 1991, De la justification, Paris, Gallimard. Gallimard.

BOLTANSKI, L. et CHIAPELLO, E. 1999, Le nouvel esprit du capitalisme, Paris,

Gazeta Wyborcza 2008, «MEN : Religią na maturze zajmie się Komisja Wspólna Rządu i Episkopatu » (Pour le Ministère de l'Education, c'est la Commission du Gouvernement et de l'Episcopat qui se chargera du problème de la religion au baccalauréat), 24/01/2008 .

BOUVIER, A. 2005, Le programme argumentativiste en sociologie et anthropologie cognitives, in RAMOGNINO, N. et VERGES, P. (dir.), Sociologie et cognition sociale, Publications de l'Univ. de Provence.

DEBRAY, R. 2002, L'enseignement du fait religieux dans l'école laïque, Paris, Odile Jacob.

DEROUET-BESSON, M.-C. 2004, «Les cents fruits d'un marronnier. Éléments pour l'histoire d'un lieu commun : l'ouverture de l'école », Éducation et Sociétés n¹3.

ELSTER, J. (dir.) 1996, The roundtable talks and the breakdown of communism, Chicago, The Univ. of Chicago Press.

Głos Nauczycielski (Revue du syndicat enseignant ZNP), 2/4/1991, 9/5/1991, 20/5/1991, Warszawa, Wyd. Głos Nauczycielski.

GRABOWSKA, M. 2008, «Jednostka i wspólnota» (L'individu et la communauté), Chrześcijaństwo, świat, polityka, Warszawa, Instytut Politologii Uniw. Wyszyńskiego.

HALASZ, G. 2002, La décentralisation dans les systèmes éducatifs de l'Europe orientale et centrale, in BAJOMI, I. et DEROUET, J.-L. (2002).

KAWALEC, K. 2008, Problemy obfitości (Des problèmes de quantité), in Prace komisji do oceny podręczników szkolnych, Tom VI, Kraków, Wydawnictwo PAU.

KONARZEWSKI, K. 2004, Reforma oświaty. Podstawa programowa $i$ warunki krztatcenia (La réforme de l'école. Les programmes scolaires et les conditions d'enseignement), Warszawa, Instytut Spraw Publicznych.

JACZYNOWSKA, M. 2006, «Kiedy urodził się Jezus »(Quand Jésus est né), Gazeta Wyborcza, 23/12/2006.

MAŁKOWSKI. T. 2000, Historia I. Podręcznik do gimnazjum (Histoire I. Manuel pour le collège), Gdańsk, Gdańskie Wyd. Oświatowe.

MROZEWICZ, L. 2002, Historia. Podrzęcznik do pierwszej klasy liceum (Histoire. Manuel pour la classe de seconde), Poznań, Nowa Era.

Nowa Szkoła 1989 (Revue du Ministère de l'Education), «Edukacja w toku obrad Okrągłego Stołu » (L'éducation pendant les discussions de la Table Ronde), 7/1989, pp 389-409 .

Nowa Szkoła 1990, «Ustawa o systemie Oświaty. Projekt » (Le projet de loi sur le système scolaire), 4/1990, pp. 452-460 .

PEZDA, A. 2007, «MEN jednak cenzuruje podręczniki » (Finalement, le Ministère de l'Éducation censure les manuels), Gazeta Wyborcza, 15/10/2007 .

PIETRACHA, M. 2001, Historia I. Podręcznik dla pierwszej klasy gimnazjum (Histoire I. Manuel pour la classe de sixième), Warszawa, Wyd. Szkolne i Pedagogiczne.

PUTKIEWICZ, E. et WIŁKOMIRSKA, A. 2004, Szkoły publiczne i niepubliczne (Les écoles publiques et non-publiques), Warszawa, Instytut Spraw Publicznych.

Przegląd Oświatowy (Revue du syndicat enseignant Solidarité), 8/4/1990, 15/5/1990, 
1/6/1990, 1/2/1991, 1/4/1991, 15/4/1991, 1/5/1991, 1/10/1991, Gdańsk, Wyd. Zarząd Regionu Gdańskiego NSZZ "Solidarność" .

ROTT, D. 2003, «Z błedami wycofujemy » (On retire ceux qui ont des fautes), Gazeta Wyborcza, 14/11/2003.

RYKOWSKI, Z. 1994, Narodziny demokratycznego systemu władzy : o Komitetach Obywatelskich w latach 1989-1992 (La naissance du système de pouvoir démocratique : sur les comités citoyens dans les années 1989-1992), in Lewenstein et Pawlik, A miało być tak pięknie, Warszawa, I.S.N.S.

TOURAINE, A. et al. 1982, Solidarité : analyse d'un mouvement social, Paris, Fayard.

VAN ZANTEN, A. et BALL, S. "Comparer pour comprendre: globalisation, réinterprétations nationales et recontextualisations locales », Revue de l'Institut de Sociologie, $\mathrm{n}^{\circ}$ 1(4), pp. 113-131. 